\title{
Erratum to: Diagnostic imaging of dementia with Lewy bodies by susceptibility-weighted imaging of nigrosomes versus striatal dopamine transporter single-photon emission computed tomography: a retrospective observational study
}

\author{
Koji Kamagata $^{1}$ - Tomoya Nakatsuka ${ }^{2}$ Ryuji Sakakibara ${ }^{3}$. Yohei Tsuyusaki ${ }^{3}$. \\ Tomohiro Takamura ${ }^{4} \cdot$ Kanako Sato $^{1} \cdot$ Michimasa Suzuki $^{1} \cdot$ Masaaki Hori $^{1}$ • \\ Kanako K Kumamaru ${ }^{1}$. Tsutomu Inaoka ${ }^{2}$. Shigeki Aoki ${ }^{1} \cdot$ Hitoshi Terada $^{2}$
}

Published online: 22 March 2017

(C) Springer-Verlag Berlin Heidelberg 2017

Erratum to: Neuroradiology

DOI: $10.1007 / \mathrm{s} 00234-016-1773-z$

The authors apologize for misinterpreting the spatial resolution in the paper by Noh et al. in the Discussion of "Diagnostic imaging of dementia with Lewy bodies by susceptibility-weighted imaging of nigrosomes versus striatal dopamine transporter singlephoton emission computed tomography: a retrospective observational study", DOI: 10.1007/s00234-016-1773-z:

The sentence should read:

In addition, the resolution of the SWI sequence used in this study was $0.9 \times 0.9 \times 2 \mathrm{~mm}$, which was slightly inferior to that used by Schwarz et al. $(0.55 \times 0.55 \times 0.7 \mathrm{~mm}$ or $0.43 \times 0.43 \times$ $0.75)$ and Noh et al. $(0.5 \times 0.5 \times 1.5 \mathrm{~mm}$, particularly at a slice thickness of $2 \mathrm{~mm}$ ).

The online version of the original article can be found at http://dx.doi. org/10.1007/s00234-016-1773-z

Koji Kamagata

kkamagat@juntendo.ac.jp

Department of Radiology, Juntendo University Graduate School of Medicine, 2-1-1 Hongo, Bunkyo-ku, Tokyo 113-8421, Japan

2 Department of Radiology, Toho University Sakura Medical Center, Sakura, Japan

3 Department of Neurology, Toho University Sakura Medical Center, Sakura, Japan

4 Department of Radiology, University of Yamanashi, Yamanashi, Japan 